\title{
Concept Study of a Radio Array Embedded in a Deep Gen2-like Optical Array
}

\section{The IceCube Gen2 Collaboration}

(a complete list of authors can be found at the end of the proceedings)

E-mail: abigail.bishop@wisc.edu

The IceCube Neutrino Observatory has discovered a diffuse astrophysical flux up to $10 \mathrm{PeV}$ and is now planning a large extension with IceCube-Gen2, including an optical array and a large radio array at shallow depth [1]. Neutrino searches for energies $>100 \mathrm{PeV}$ are best done with such shallow radio detectors like the Askaryan Radio Array (ARA) or similar (buried as deep as 200 meters below the surface) as they are cheaper to deploy. This poster explores the potential of opportunistically burying radio antennas within the planned IceCube-Gen2 detector volume (between 1350 meters and 2600 meters below the surface). A hybrid detection of events in optical and radio could substantially improve the uncertainty of neutrino cascade direction as radio signals do not scatter in ice. We show the first results of simulating neutrinos from an astrophysical and a cosmogenic flux interacting with 9760 ARA-style vertically polarized radio antennas distributed evenly across 122 strings.

Corresponding authors: Abby Bishop ${ }^{1 *}, \mathrm{Lu} \mathrm{Lu}^{1}$, Albrecht Karle ${ }^{1}$, Ben Hokanson-Fasig ${ }^{1}$

${ }^{1}$ University of Wisconsin - Madison

* Presenter

$37^{\text {th }}$ International Cosmic Ray Conference (ICRC 2021)

July 12th-23rd, 2021

Online - Berlin, Germany 


\section{Introduction}

IceCube has detected a diffuse cosmic neutrino flux in the energy range from $60 \mathrm{TeV}$ to more than $10 \mathrm{PeV}$ [2-5]. IceCube-Gen2 will substantially improve the event rates especially for cascade events. IceCube-Gen2 will include a deep optical array of about $8 \mathrm{~km}^{3}$ instrumented volume and the Gen2-Radio array for high energy ( $>30 \mathrm{PeV})$ neutrino observations [1]. The Gen2-Radio array is designed using insights from previous radio neutrino experiments such as RICE [6], ANITA [7], ARA [8, 9], ARIANNA [10, 11], and RNOG (currently in construction in Greenland) [12]. The Gen2-radio array will achieve a sensitivity at high energies (above $30 \mathrm{PeV}$ ) cosmic neutrino flux by more than an order of magnitude of what would be imaginable with an optical array. Here, we are investigating the possibility to enhance the performance of the Gen2 optical array in the range from a few $\mathrm{PeV}$ to above $100 \mathrm{PeV}$ by co-deploying radio antennas together with the optical sensors, one antenna for each optical module. In this energy range, Gen2 will over the time space of a decade, observe more than 50 neutrino induced cascade events above $10 \mathrm{PeV}$. Useful experiences exist from the deployment of AURA (Askaryan Under Ice Radio Array, [13] a prototype radio detector that was co-deployed with IceCube.

Could the additional radio data improve Gen2-Optical measurements or help connect flux measurements between $3 \mathrm{PeV}$ and $100 \mathrm{PeV}$ ? The considered radio component is not a stand alone detector for detecting ultrahigh energy neutrinos. It primarily would be an augmentation of optical array which would provide all triggering.

The results indicate that a substantial amount of instrumentation would be required. Initial considerations suggest that the implementation in the Gen 2 architecture would be quite straightforward. The basic configuration is that one antenna would be hooked up with every optical module. The instrumentation showcased here is not planned for Gen2, however the reader may find characteristics of such a configuration, studied here for the first time, to be intriguing.

\section{Simulation and detector configuration}

The simulation package "Python for Radio Experiments" (PyREx) ${ }^{1}$ was used to simulate 40,000 neutrino events at select energies ranging from $1 \mathrm{PeV}$ to $10 \mathrm{EeV}$. Neutrino interactions are simulated using the CTW model described in [14]. The following neutrino properties are determined randomly according to the cosmogenic model: every flavor is equally possible and the probability that a particle is a neutrino (as opposed to an anti-neutrino) is $0.78,0.61$, and 0.61 for electron-, muon- and tau neutrino respectively [15]. After interacting, the simulation propagates Askaryan signals to all antennas following the Alvarez-Muniz, Romero-Wolf, Zas parameterization then simulates their responses [16].

The radio detector component is conceived such that that every optical sensor on the string, there is one radio antenna associated with that sensor and located $8 \mathrm{~m}$ above it. Thus, a simulation was performed with 9760 ARA-style vertically polarized (Vpol) radio antennas located on the 120 Gen2 Strings, 80 per string, between 1350 and 2600 meters below the Antarctic ice's surface. The simulation assumed a vertical spacing of antennas is $17 \mathrm{~m}$ and the spacing between strings is $\approx 240 \mathrm{~m}$. The technical implementation would be such that every multi-PMT optical sensor would

${ }^{1}$ Github: https://github.com/bhokansonfasig/pyrex 
feature one additional readout channel that would connect an envelope detector to the antenna, which is located $7 \mathrm{~m}$ above the DOM in the null of the antenna.

In the simulation 40,000 events are generated and simulated at select energies using the radiosignal simulation software PyREx and the aforementioned parameters. Next, all events with $6 \mathrm{PeV}$, $10 \mathrm{PeV}, 30 \mathrm{PeV}$, or $100 \mathrm{PeV}$ of energy and located within 300 meters of IceCube-Gen 2 boundary are isolated and processed using the optical-signal simulation software IceTray ${ }^{2}(\approx 6,000$ events for each energy). We primarily investigate a mode of operation in which the triggering would be provided entirely by the optical sensors. Thus, the Gen2 optical sensors would provide both the trigger for the readout and the readout channel for the RF signal. It would be realized as an additional channel to the 16 or 18 PMT channels in consideration for Gen2 [17]. This trigger and readout would be quite straightforward to implement as the formation of the optical trigger is managed with the Gen2 optical trigger system. The readout decision is made at the DOM level and does not require a global event trigger formation.

Considerations of RF noise from nearby optical sensors will be ignored for this conceptual study, but would need to be considered if this topic is studied further. Based on previous measurements with the AURA detectors [13], it is assumed this would not be a major issue.

\subsection{Optical-Radio Hybrid Events}

Radio waves travel essentially unscattered in ice, unlike the optical signal. Therefore the radio signals could substantially improve angular reconstruction of events, especially for cascade-like events where the angular resolution of the optical detector is more limited. In some cases, it could also add substantial information to events with the vertex outside of the array. The primary energy range of events in consideration is from a few $\mathrm{PeV}$ to $100 \mathrm{PeV}$ - events with bright optical signals in the optical detector.

To investigate a possible coincident detection of radio and optical signals, we start with the detailed examination of an event of $30 \mathrm{PeV}$ energy and travelling downwards at a zenith angle of 65 degrees. The event is simulated in both PyREx and IceCube's Gen2-Optical IceTray simulation to simulate both the radio and the optical response. The detector response can be seen in Figure 1 where the the detected event topology is shown for optical in the left panel and for RF in the right panel where the cone of coherent emission is clearly visible.

The optical and RF signal waveforms are shown in Figure 2 for one location. The short impulsive radio signal is shown on the same time scale as the optical signal which extends over several $100 \mathrm{~ns}$. The time resolution of radio signal detection would be at the level of a few ns, limited only the system clock synchronization. The optical trigger for every module would be at the level of a few photo-electrons. As mentioned, the readout of every antenna would be initiated and performed by the local DOM. In the right panel we show three waveforms recorded on consecutive antennas on a string at a substantial distance of 350 to $367 \mathrm{~m}$. The vertical spacing of the antennas is $16 \mathrm{~m}$. The amplitude profile of the three signals will provide very good tool to locate the intersection of the radio cone with the Gen2-string to a precision small compared to the antenna spacing.

\footnotetext{
${ }^{2}$ Github: https://github.com/icecube/icetray/tree/gen2-optical-sim
} 

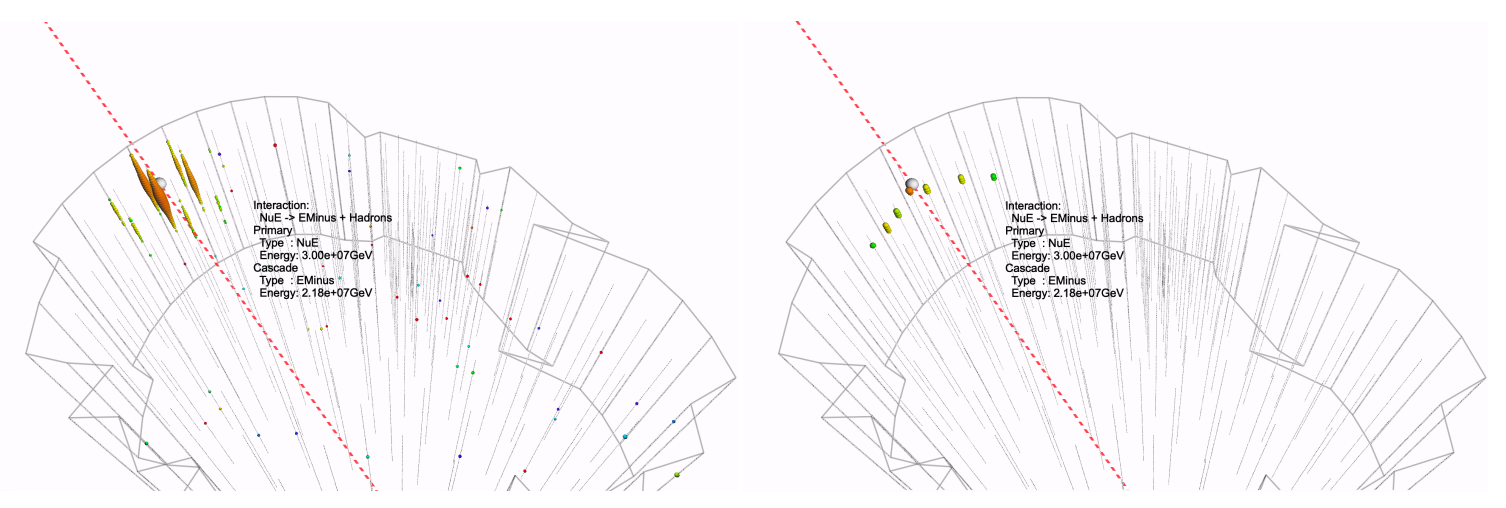

Figure 1: Image of the detector response by the Gen2-Optical detector and the hypothetical radio detector for a neutrino event that can be observed perfectly by both detectors. The size of each bubble corresponds to the strength of the received signal at the corresponding radio antenna or DOM, while the color corresponds to the time the signal peaked.
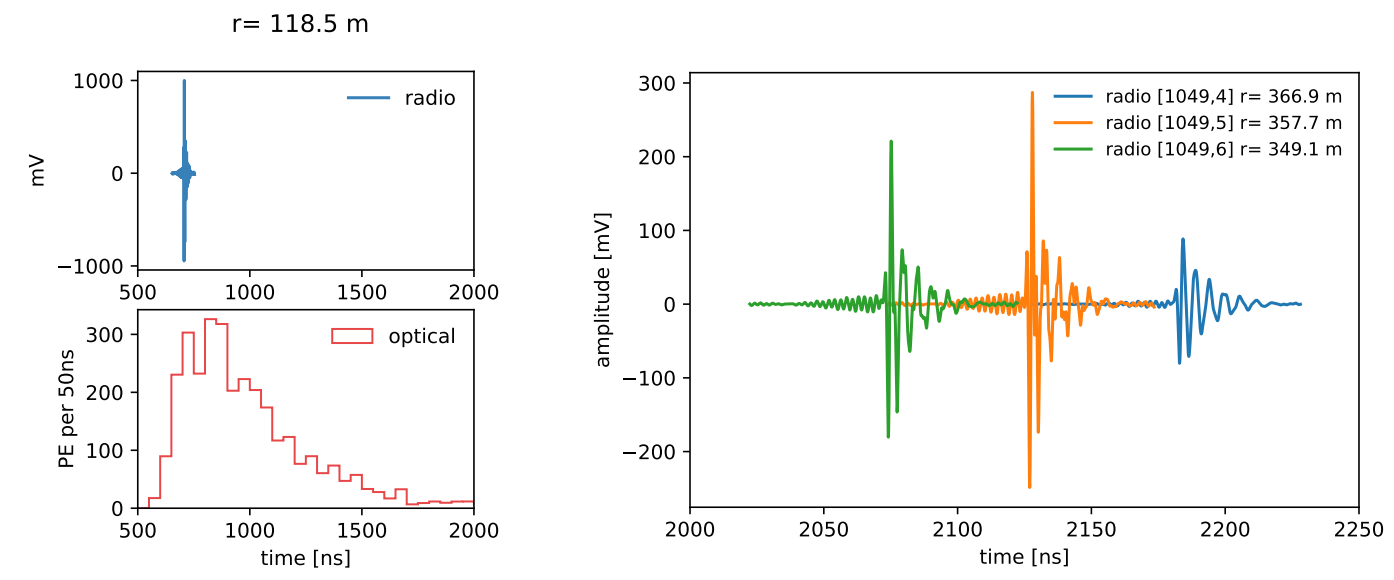

Figure 2: The simulated optical and RF waveforms are shown for the same neutrino event on the same time scale. The optical sensor with the RF extension is located at a distance of $118.5 \mathrm{~m}$ from the vertex. In the right panel three waveforms are shown for a string at a distance of $350 \mathrm{~m}$.

\subsection{Event reconstruction}

Although angular reconstruction techniques are still in-progress, the benefits of radio detectors locating these neutrinos are easy to picture from the separation of the radio and optical signals and the informative topology of the radio event, especially for cascades. The waveforms of photons from a PeV event in the optical extend typically for hundreds of nanoseconds, while the RF time resolution would be a few nanoseconds.

It is important to recall that every event that will be considered will have a very bright optical signal of typically more than 100,000 photoelectrons. Thus the optical reconstruction will provide a good constraint on the vertex and a reasonable prior on the direction. Thus even a few relatively faint radio signals can contribute significantly to the reconstruction. For this reason, we consider a signal above $2 \sigma$ in at least 2 antennas to be a threshold for hybrid events. 
One strategy of event reconstruction uses the recorded RF signals to locate the Radio Cherenkov cone. In this reconstruction we use the vertex as a given. The vertex can be determined in the optical channel to a precision of about $20 \mathrm{~m}$. The RF channel will allow a better vertex position of easily $10 \mathrm{~m}$. However the timing signals do not provide the direction. Instead we use the presence of RF signals as an indicator for the proximity of the Cherenkov cone. In a most basic version, we consider the brightest antenna signal on a string to be exactly on cone. Every antenna with a hit provides a constraint to the neutrino direction as illustrated in Figure 3. In the case of the simulated $30 \mathrm{PeV}$ cascade there is a signal on 7 strings, each of which provides one contour. The intersection of the contours indicates the direction of the event, and the differences of the intersections indicate a measure of the uncertainty. The convex hull points to a resolution of better than 1 degree. Simple optimizations of this reconstruction would include to use the amplitude of the antenna signals and their distance from the vertex as a weight in this procedure. For example, here we used only the waveform with the largest amplitude in Figure 2. An obvious improvement would be fit the amplitude profile of the Askaryan cone, or simply take some weighted average as a measure of the RF Cherenkov cone. For an event like the one investigated a resolution of a fraction of a degree is well conceivable.
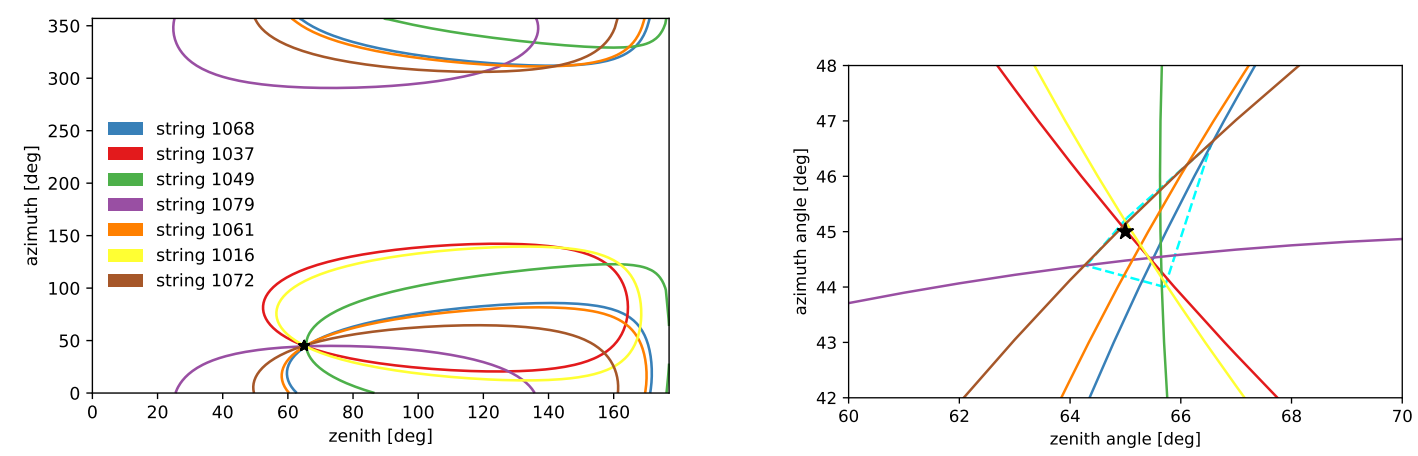

Figure 3: A simple event reconstruction leads to one contour for every antenna signal. Here the solutions for the brightest antenna signal on each string is shown. The intersection indicates the solution. The close-up in the right panel indicates a convex hull of the intersections, which may be considered as an estimate of the uncertainty of less than 1 degree. The true position of the event is: zenith $=65^{\circ}$ and azimuth $=45^{\circ}$. The resolution would significantly improve the Gen2 optical cascade reconstruction with a requirement of 10 degrees.

\subsection{Event rates}

Since the focus of this investigation is on cascade events with possible RF content this analysis is based on simulations of electron and electron-anti neutrinos. The threshold for the optical channel is set sufficiently high to include only bright events at PeV energy scale. Every event at the energies considered $(6,10,30$ and $100 \mathrm{PeV})$ will produce a very bright signal in the optical detector.

Figure 4 shows event rates for an injected IceCube neutrino flux of $\mathrm{E}^{-2.49}$ using high energy starting event (HESE) [2] and PeV energy partially contained events (PEPE) data [5]. In this figure, we assume and effective volume for detection cascade events to be 10 times that of IceCube, in line 




Figure 4: Number of Glashow hadronic, charged current and neutral current events from electron (anti)neutrinos selected by HESE [2] and PEPE [5] analyses assuming a single power law flux of $\mathrm{E}^{-2.49}$ for 10 years of IceCube livetime. Numbers are multiplied by ten to scale up to the Gen2 event rate (which is approximately 10 times larger in instrumented volume than IceCube).

with the ratio of the instrumented volumes of Gen2 and IceCube. For a livetime of 10 years we obtain a total of 70 cascade events in the neutrino energy range from $5 \mathrm{PeV}$ to $100 \mathrm{PeV}$, which includes an important contribution of 54 events from the Glashow resonance. This channel is especially intriguing, as it will provide an ensemble of electron antineutrinos at sub-degree resolution. We then ask the question, what is the fraction of events that is seen in the optical that will also be seen in RF.

For that we determine the effective volume of such hybrid radio and optical configuration with signals in the RF channel. The effective volume may be seen in Figure 5 for three threshold conditions in the RF channels. One could imagine to use antennas more optimized for the purpose and taking advantage of the larger hole size. A gain in effective height of the antenna of a factor of 2 or 3 seems plausible. This is in part the reason why we show results with several thresholds, including 2 and 1 sigma on 2 antennas. For illustration we show the effective volume for three threshold settings in the RF.

Table 1 shows the estimated fraction of events that also record an RF signal, ranging from about $40 \%$ at $10 \mathrm{PeV}$ to above $90 \%$ at $100 \mathrm{PeV}$. The total number of events with RF content would be in the range of 5 to 10 events.

The option to deploy antenna arrays with self trigger capability was not investigated. The implementation approach would be very different. Phased arrays of antennas would be installed primarily on the outer strings of the array, and primarily at shallower depth above $1500 \mathrm{~m}$ where the coldest ice is located. An initial estimate indicates that the effective volume at very high energies ( $>1 \mathrm{EeV}$ ) would be substantially larger. Such an approach would be intriguing but the technical realization would be a sizeable project, despite the "free" boreholes. 


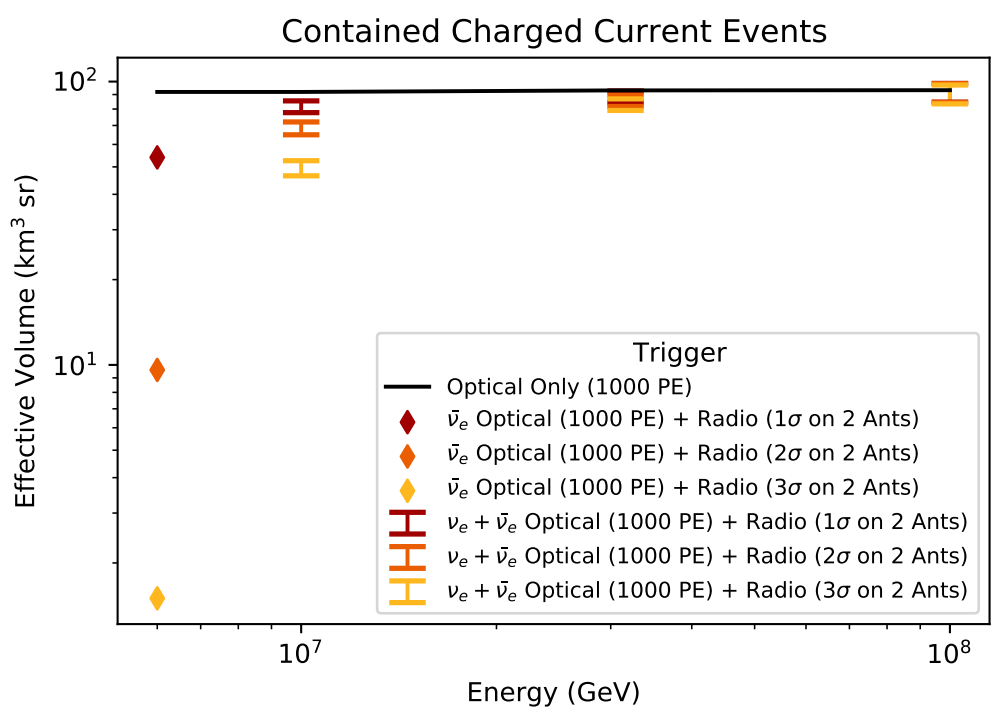

Figure 5: Effective volumes are shown for electron neutrino induced cascade events that trigger both Gen2Optical and have a signal in the hypothetical radio detector for varying radio trigger requirements. Also indicated is a the geometric volume of the Gen2 optical array that is fully effective for the set condition at all energies.

\begin{tabular}{|c||c|}
\hline Energy & Fraction of events with RF content \\
\hline \hline $6 \mathrm{PeV}$ & 0.11 \\
$10 \mathrm{PeV}$ & 0.41 \\
$30 \mathrm{PeV}$ & 0.84 \\
$100 \mathrm{PeV}$ & 0.91 \\
\hline
\end{tabular}

Table 1: The fraction of contained events with RF content are shown. The requirement for the RF channel is at least 2 sigma on 2 antennas.

\section{Summary}

At the highest energies due to Earth absorption most of the arriving neutrinos are from the southern sky, making it difficult to reject cosmic-ray background except for starting events. The cascade channel is the main contributor to probe Universe beyond multi-PeVs. A study was performed of a Gen2 style detector configuration that is augmented with vertically polarized antennas, one antenna connected to every optical sensor. A complete hybrid RF/optical simulation was performed of such volume detector. At energies above a few PeV the events are starting to contain some RF signals, at $10 \mathrm{PeV}$ the efficiency is about 50\%. Even a very coarse attempt to use the RF information for reconstruction shows that the directional reconstruction could improve substantially to the range of 1 degree and possibly well below. For the simulations only contained events were used. The addition of events with vertex outside the array but still with a bright optical signal (> 1000 photoelectrons) may increase the effective volume slightly. In addition to the multimessenger opportunities that would be made available by embedding radio antennas in the IceCube 
detector, the additional radio data may also benefit signal/background separation of non-contained events pointing towards the detector. However the overall rates with the chosen configuration are modest. For the choice of antenna, events below $3 \mathrm{PeV}$ would rarely have an RF signal. The option to deploy antenna arrays with self trigger capability was not investigated. An initial estimate indicates that the effective volume at very high energies ( $>1 \mathrm{EeV}$ ) would be an order of magnitude larger. However it would be still short of the science goals of Gen2 and be significantly smaller than the planned Gen2 radio array.

\section{References}

[1] IceCube Collaboration Journal of Physics G: Nuclear and Particle Physics 48 no. 6, (Apr, 2021) 060501.

[2] IceCube Collaboration Science 342 no. 6161, (2013) .

[3] IceCube Collaboration The Astrophysical Journal 833 no. 1, (Dec, 2016) 3.

[4] IceCube Collaboration Phys. Rev. Lett. 125 (Sep, 2020) 121104.

[5] IceCube Collaboration Nature 591 no. 7849, (Mar, 2021) 220-224.

[6] I. Kravchenko, C. Cooley, S. Hussain, and et al. Physical Review D 73 no. 8, (Apr, 2006) .

[7] P. Gorham, P. Allison, O. Banerjee, and et al. Physical Review D 98 no. 2, (Jul, 2018) .

[8] ARA Collaboration Astroparticle Physics 35 no. 7, (Feb., 2012) 457-477.

[9] ARA Collaboration Physical Review D 102 no. 4, (Aug, 2020).

[10] A. Anker, S. Barwick, H. Bernhoff, and et al. Journal of Cosmology and Astroparticle Physics 2020 no. 03, (Mar, 2020) 053-053.

[11] A. Anker, S. Barwick, H. Bernhoff, and et al. Advances in Space Research 64 no. 12, (Dec, 2019) 2595-2609.

[12] J. Aguilar, P. Allison, J. Beatty, and et al. Journal of Instrumentation 16 no. 03, (Mar, 2021) P03025.

[13] H. Landsman, L. Ruckman, and G. Varner Nuclear Instruments and Methods in Physics Research Section A: Accelerators, Spectrometers, Detectors and Associated Equipment 604 no. 1, Supplement, (2009) S70-S75. ARENA 2008.

[14] A. Connolly, R. S. Thorne, and D. Waters Physical Review D 83 no. 11, (Jun, 2011) .

[15] A. Bhattacharya, R. Gandhi, W. Rodejohann, and A. Watanabe Journal of Cosmology and Astroparticle Physics 2011 no. 10, (Oct, 2011) 017-017.

[16] J. Alvarez-Muñiz, A. Romero-Wolf, and E. Zas Physical Review D 84 no. 10, (Nov, 2011) .

[17] IceCube Gen2 Collaboration PoS ICRC2021 (these proceedings) 1062. 


\section{Full Author List: IceCube-Gen2 Collaboration}

R. Abbasi ${ }^{17}$, M. Ackermann ${ }^{71}$, J. Adams ${ }^{22}$, J. A. Aguilar ${ }^{12}$, M. Ahlers ${ }^{26}$, M. Ahrens ${ }^{60}$, C. Alispach ${ }^{32}$, P. Allison ${ }^{24,25}$, A. A. Alves $\mathrm{Jr}^{35}$, N. M. Amin ${ }^{50}$, R. An ${ }^{14}$, K. Andeen ${ }^{48}$, T. Anderson ${ }^{67}$, G. Anton ${ }^{30}$, C. Argüelles ${ }^{14}$, T. C. Arlen ${ }^{67}$, Y. Ashida ${ }^{45}$, S. Axani ${ }^{15}, \mathrm{X}$. Bai $^{56}$, A. Balagopal V. ${ }^{45}$, A. Barbano ${ }^{32}$, I. Bartos ${ }^{52}$, S. W. Barwick ${ }^{34}$, B. Bastian ${ }^{71}$, V. Basu ${ }^{45}$, S. Baur ${ }^{12}$, R. Bay ${ }^{8}$, J. J. Beatty ${ }^{24,}$ 25, $^{25}$, K.-H. Becker ${ }^{70}$, J. Becker Tjus ${ }^{11}$, C. Bellenghi ${ }^{31}$, S. BenZvi ${ }^{58}$, D. Berley ${ }^{23}$, E. Bernardini ${ }^{71}$, 72, D. Z. Besson ${ }^{38,73}$, G. Binder ${ }^{8,9}$, D. Bindig $^{70}$, A. Bishop ${ }^{45}$, E. Blaufuss ${ }^{23}$, S. Blot ${ }^{71}$, M. Boddenberg ${ }^{1}$, M. Bohmer ${ }^{31}$, F. Bontempo ${ }^{35}$, J. Borowka ${ }^{1}$, S. Böser ${ }^{46}$, O. Botner ${ }^{69}$, J. Böttcher ${ }^{1}$, E. Bourbeau ${ }^{26}$, F. Bradascio ${ }^{71}$, J. Braun ${ }^{45}$, S. Bron ${ }^{32}$, J. Brostean-Kaiser ${ }^{71}$, S. Browne ${ }^{36}$, A. Burgman ${ }^{69}$, R. T. Burley ${ }^{2}$, R. S. Busse ${ }^{49}$, M. A. Campana ${ }^{55}$, E. G. Carnie-Bronca ${ }^{2}$, M. Cataldo ${ }^{30}$, C. Chen ${ }^{6}$, D. Chirkin ${ }^{45}$, K. Choi ${ }^{62}$, B. A. Clark ${ }^{28}$, K. Clark ${ }^{37}$, R. Clark $^{40}$, L. Classen ${ }^{49}$, A. Coleman ${ }^{50}$, G. H. Collin ${ }^{15}$, A. Connolly ${ }^{24}$, 25 , J. M. Conrad ${ }^{15}$, P. Coppin ${ }^{13}$, P. Correa ${ }^{13}$, D. F. Cowen ${ }^{66,67}$, R. $\mathrm{Cross}^{58}$, C. Dappen ${ }^{1}$, P. Dave ${ }^{6}$, C. Deaconu ${ }^{20,21}$, C. De Clercq ${ }^{13}$, S. De Kockere ${ }^{13}$, J. J. DeLaunay ${ }^{67}$, H. Dembinski ${ }^{50}$, K. Deoskar ${ }^{60}$, S. De Ridder ${ }^{33}$, A. Desai ${ }^{45}$, P. Desiati ${ }^{45}$, K. D. de Vries ${ }^{13}$, G. de Wasseige ${ }^{13}$, M. de With ${ }^{10}$, T. DeYoung ${ }^{28}$, S. Dharani ${ }^{1}$, A. Diaz $^{15}$, J. C. Díaz-Vélez ${ }^{45}$, M. Dittmer ${ }^{49}$, H. Dujmovic ${ }^{35}$, M. Dunkman ${ }^{67}$, M. A. DuVernois ${ }^{45}$, E. Dvorak ${ }^{56}$, T. Ehrhardt ${ }^{46}$, P. Eller ${ }^{31}$, R. Enge $^{35,36}$, H. Erpenbeck ${ }^{1}$, J. Evans ${ }^{23}$, J. J. Evans ${ }^{47}$, P. A. Evenson ${ }^{50}$, K. L. Fan ${ }^{23}$, K. Farrag ${ }^{41}$, A. R. Fazely ${ }^{7}$, S. Fiedlschuster ${ }^{30}$, A. T. Fienberg ${ }^{67}$, K. Filimonov ${ }^{8}$, C. Finley ${ }^{60}$, L. Fischer ${ }^{71}$, D. Fox ${ }^{66}$, A. Franckowiak ${ }^{11,71}$, E. Friedman ${ }^{23}$, A. Fritz ${ }^{46}$, P. Fürst ${ }^{1}$, T. K. Gaisser ${ }^{50}$, J. Gallagher ${ }^{44}$, E. Ganster ${ }^{1}$, A. Garcia ${ }^{14}$, S. Garrappa ${ }^{71}$, A. Gartner ${ }^{31}$, L. Gerhardt ${ }^{9}$, R. Gernhaeuser ${ }^{31}$, A. Ghadimi ${ }^{65}$, P. Giri ${ }^{39}$, C. Glaser ${ }^{69}$, T. Glauch ${ }^{31}$, T. Glüsenkamp ${ }^{30}$, A. Goldschmidt ${ }^{9}$, J. G. Gonzalez ${ }^{50}$, S. Goswami ${ }^{65}$, D. Grant ${ }^{28}$, T. Grégoire ${ }^{67}$, S. Griswold ${ }^{58}$, M. Gündüz ${ }^{11}$, C. Günther ${ }^{1}$, C. Haack ${ }^{31}$, A. Hallgren ${ }^{69}$, R. Halliday ${ }^{28}$, S. Hallmann ${ }^{71}$, L. Halve ${ }^{1}$, F. Halzen ${ }^{45}$, M. $\mathrm{Ha} \mathrm{Minh}^{31}$, K. Hanson ${ }^{45}$, J. Hardin ${ }^{45}$, A. A. Harnisch ${ }^{28}$, J. Haugen ${ }^{45}$, A. Haungs ${ }^{35}$, S. Hauser ${ }^{1}$, D. Hebecker ${ }^{10}$, D. Heinen ${ }^{1}$, K.

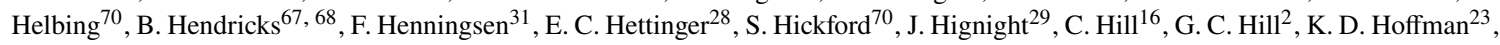
B. Hoffmann ${ }^{35}$, R. Hoffmann ${ }^{70}$, T. Hoinka ${ }^{27}$, B. Hokanson-Fasig ${ }^{45}$, K. Holzapfel ${ }^{31}$, K. Hoshina ${ }^{45,64}$, F. Huang ${ }^{67}$, M. Huber ${ }^{31}$, T.

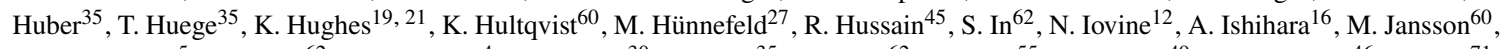
G. S. Japaridze ${ }^{5}$, M. Jeong ${ }^{62}$, B. J. P. Jones ${ }^{4}$, O. Kalekin ${ }^{30}$, D. Kang ${ }^{35}$, W. Kang ${ }^{62}$, X. Kang ${ }^{55}$, A. Kappes ${ }^{49}$, D. Kappesser ${ }^{46}$, T. Karg ${ }^{71}$, M. Karl ${ }^{31}$, A. Karle ${ }^{45}$, T. Katori ${ }^{40}$, U. Katz ${ }^{30}$, M. Kauer ${ }^{45}$, A. Keivani ${ }^{52}$, M. Kellermann ${ }^{1}$, J. L. Kelley ${ }^{45}$, A. Kheirandish ${ }^{67}$, K. Kin ${ }^{16}$, T. Kintscher ${ }^{71}$, J. Kiryluk ${ }^{61}$, S. R. Klein ${ }^{8,9}$, R. Koirala ${ }^{50}$, H. Kolanoski ${ }^{10}$, T. Kontrimas ${ }^{31}$, L. Köpke ${ }^{46}$, C. Kopper ${ }^{28}$, S. Kopper ${ }^{65}$, D. J. Koskinen ${ }^{26}$, P. Koundal ${ }^{35}$, M. Kovacevich ${ }^{55}$, M. Kowalski ${ }^{10,71}$, T. Kozynets ${ }^{26}$, C. B. Krauss ${ }^{29}$, I. Kravchenko ${ }^{39}$, R. Krebs ${ }^{67}{ }^{68}$, E.

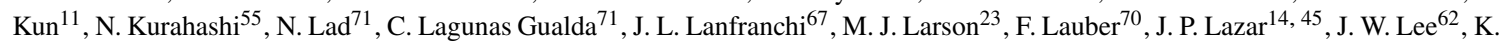

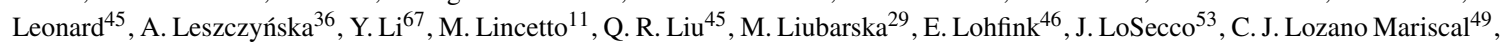
L. $\mathrm{Lu}^{45}$, F. Lucarelli ${ }^{32}$, A. Ludwig ${ }^{28,42}$, W. Luszczak ${ }^{45}$, Y. Lyu ${ }^{8,9}$, W. Y. Ma ${ }^{71}$, J. Madsen ${ }^{45}$, K. B. M. Mahn ${ }^{28}$, Y. Makino ${ }^{45}$, S. Mancina $^{45}$, S. Mandalia ${ }^{41}$, I. C. Mariş ${ }^{12}$, S. Marka ${ }^{52}$, Z. Marka ${ }^{52}$, R. Maruyama ${ }^{51}$, K. Mase ${ }^{16}$, T. McElroy ${ }^{29}$, F. McNally ${ }^{43}$, J. V. Mead $^{26}$, K. Meagher ${ }^{45}$, A. Medina ${ }^{25}$, M. Meier ${ }^{16}$, S. Meighen-Berger ${ }^{31}$, Z. Meyers ${ }^{71}$, J. Micallef ${ }^{28}$, D. Mocklerr ${ }^{12}$, T. Montaruli ${ }^{32}$, R. W. Moore ${ }^{29}$, R. Morse ${ }^{45}$, M. Moulai ${ }^{15}$, R. Naab ${ }^{71}$, R. Nagai ${ }^{16}$, U. Naumann ${ }^{70}$, J. Necker ${ }^{71}$, A. Nelles ${ }^{30,71}$, L. V. Nguyễn ${ }^{28}$, H. Niederhausen $^{31}$, M. U. Nisa ${ }^{28}$, S. C. Nowicki ${ }^{28}$, D. R. Nygren ${ }^{9}$, E. Oberla ${ }^{20,21}$, A. Obertacke Pollmann ${ }^{70}$, M. Oehler ${ }^{35}$, A. Olivas ${ }^{23}$, A. Omeliukh ${ }^{71}$, E. O' $\operatorname{Sullivan}^{69}$, H. Pandya ${ }^{50}$, D. V. Pankova ${ }^{67}$, L. Papp ${ }^{31}$, N. Park ${ }^{37}$, G. K. Parker ${ }^{4}$, E. N. Paudel ${ }^{50}$, L. Paul ${ }^{48}$, C. Pérez de $\operatorname{los} \operatorname{Heros}^{69}$, L. Peters ${ }^{1}$, T. C. Petersen ${ }^{26}$, J. Peterson ${ }^{45}$, S. Philippen ${ }^{1}$, D. Pieloth ${ }^{27}$, S. Pieper ${ }^{70}$, J. L. Pinfold ${ }^{29}$, M. Pittermann ${ }^{36}$, A. Pizzuto $^{45}$, I. Plaisier ${ }^{71}$, M. Plum ${ }^{48}$, Y. Popovych ${ }^{46}$, A. Porcelli ${ }^{33}$, M. Prado Rodriguez ${ }^{45}$, P. B. Price ${ }^{8}$, B. Pries ${ }^{28}$, G. T. Przybylski ${ }^{9}$, L. Pyras $^{71}$, C. Raab ${ }^{12}$, A. Raissi ${ }^{22}$, M. Rameez ${ }^{26}$, K. Rawlins ${ }^{3}$, I. C. Rea ${ }^{31}$, A. Rehman ${ }^{50}$, P. Reichherzer ${ }^{11}$, R. Reimann ${ }^{1}$, G. Renzi ${ }^{12}$, E. Resconi ${ }^{31}$, S. Reusch ${ }^{71}$, W. Rhode 27 , M. Richman ${ }^{55}$, B. Riedel ${ }^{45}$, M. Riegel ${ }^{35}$, E. J. Roberts ${ }^{2}$, S. Robertson ${ }^{8,9}$, G. Roellinghoff ${ }^{62}$, M. Rongen ${ }^{46}$, C. Rott ${ }^{59}$ 62, T. Ruhe ${ }^{27}$, D. Ryckbosch ${ }^{33}$, D. Rysewyk Cantu ${ }^{28}$, I. Safa ${ }^{14,45}$, J. Saffer ${ }^{36}$, S. E. Sanchez Herrera ${ }^{28}$, A. Sandrock $^{27}$, J. Sandroos ${ }^{46}$, P. Sandstrom ${ }^{45}$, M. Santander ${ }^{65}$, S. Sarkar ${ }^{54}$, S. Sarkar ${ }^{29}$, K. Satalecka ${ }^{71}$, M. Scharf ${ }^{1}$, M. Schaufel ${ }^{1}$, H. Schieler $^{35}$, S. Schindler ${ }^{30}$, P. Schlunder ${ }^{27}$, T. Schmidt ${ }^{23}$, A. Schneider ${ }^{45}$, J. Schneider ${ }^{30}$, F. G. Schröder ${ }^{35}$, 50, L. Schumacher ${ }^{31}$, G. Schwefer $^{1}$, S. Sclafani ${ }^{55}$, D. Seckel ${ }^{50}$, S. Seunarine ${ }^{57}$, M. H. Shaevitz ${ }^{52}$, A. Sharma ${ }^{69}$, S. Shefali ${ }^{36}$, M. Silva ${ }^{45}$, B. Skrzypek ${ }^{14}$, D. Smith $^{19,21}$, B. Smithers ${ }^{4}$, R. Snihur ${ }^{45}$, J. Soedingrekso ${ }^{27}$, D. Soldin ${ }^{50}$, S. Söldner-Rembold ${ }^{47}$, D. Southall ${ }^{19,21}$, C. Spannfellner ${ }^{31}$, G. M. Spiczak ${ }^{57}$, C. Spiering ${ }^{71}$, 73, J. Stachurska ${ }^{71}$, M. Stamatikos ${ }^{25}$, T. Stanev ${ }^{50}$, R. Stein ${ }^{71}$, J. Stettner ${ }^{1}$, A. Steuer ${ }^{46}$, T. Stezelberger ${ }^{9}$, T.

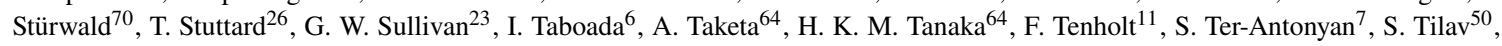
F. Tischbein ${ }^{1}$, K. Tollefson ${ }^{28}$, L. Tomankova ${ }^{11}$, C. Tönnis ${ }^{63}$, J. Torres ${ }^{24,25}$, S. Toscano ${ }^{12}$, D. Tosi ${ }^{45}$, A. Trettin ${ }^{71}$, M. Tselengidou ${ }^{30}$, C. F. Tung ${ }^{6}$, A. Turcati $^{31}$, R. Turcotte ${ }^{35}$, C. F. Turley ${ }^{67}$, J. P. Twagirayezu ${ }^{28}$, B. Ty ${ }^{45}$, M. A. Unland Elorrieta ${ }^{49}$, N. Valtonen-Mattila ${ }^{69}$, J. Vandenbroucke ${ }^{45}$, N. van Eijndhoven ${ }^{13}$, D. Vannerom ${ }^{15}$, J. van Santen $^{71}$, D. Veberic ${ }^{35}$, S. Verpoest ${ }^{33}$, A. Vieregg ${ }^{18,}$ 19, 20, 21, M. Vraeghe $^{33}$, C. Walck ${ }^{60}$, T. B. Watson ${ }^{4}$, C. Weaver ${ }^{28}$, P. Weigel ${ }^{15}$, A. Weindl ${ }^{35}$, L. Weinstock ${ }^{1}$, M. J. Weiss ${ }^{67}$, J. Weldert ${ }^{46}$, C. Welling ${ }^{71}$, C. Wendt ${ }^{45}$, J. Werthebach ${ }^{27}$, M. Weyrauch ${ }^{36}$, N. Whitehorn ${ }^{28,}{ }^{42}$, C. H. Wiebusch ${ }^{1}$, D. R. Williams ${ }^{65}$, S. Wissel ${ }^{66,67,68}$, M. Wolf $^{31}$, K. Woschnagg ${ }^{8}$, G. Wrede ${ }^{30}$, S. Wren ${ }^{47}$, J. Wulff ${ }^{11}$, X. W. Xu ${ }^{7}$, Y. Xu ${ }^{61}$, J. P. Yanez ${ }^{29}$, S. Yoshida ${ }^{16}$, S. Yu ${ }^{28}$, T. Yuan ${ }^{45}$, Z. Zhang ${ }^{61}$, S. Zierke ${ }^{1}$

\footnotetext{
${ }^{1}$ III. Physikalisches Institut, RWTH Aachen University, D-52056 Aachen, Germany

${ }^{2}$ Department of Physics, University of Adelaide, Adelaide, 5005, Australia

${ }^{3}$ Dept. of Physics and Astronomy, University of Alaska Anchorage, 3211 Providence Dr., Anchorage, AK 99508, USA

${ }^{4}$ Dept. of Physics, University of Texas at Arlington, 502 Yates St., Science Hall Rm 108, Box 19059, Arlington, TX 76019, USA

${ }^{5}$ CTSPS, Clark-Atlanta University, Atlanta, GA 30314, USA

${ }^{6}$ School of Physics and Center for Relativistic Astrophysics, Georgia Institute of Technology, Atlanta, GA 30332, USA

${ }^{7}$ Dept. of Physics, Southern University, Baton Rouge, LA 70813, USA

${ }^{8}$ Dept. of Physics, University of California, Berkeley, CA 94720, USA
} 
${ }^{9}$ Lawrence Berkeley National Laboratory, Berkeley, CA 94720, USA

${ }^{10}$ Institut für Physik, Humboldt-Universität zu Berlin, D-12489 Berlin, Germany

${ }^{11}$ Fakultät für Physik \& Astronomie, Ruhr-Universität Bochum, D-44780 Bochum, Germany

${ }^{12}$ Université Libre de Bruxelles, Science Faculty CP230, B-1050 Brussels, Belgium

${ }^{13}$ Vrije Universiteit Brussel (VUB), Dienst ELEM, B-1050 Brussels, Belgium

${ }^{14}$ Department of Physics and Laboratory for Particle Physics and Cosmology, Harvard University, Cambridge, MA 02138, USA

${ }^{15}$ Dept. of Physics, Massachusetts Institute of Technology, Cambridge, MA 02139, USA

${ }^{16}$ Dept. of Physics and Institute for Global Prominent Research, Chiba University, Chiba 263-8522, Japan

${ }^{17}$ Department of Physics, Loyola University Chicago, Chicago, IL 60660, USA

${ }^{18}$ Dept. of Astronomy and Astrophysics, University of Chicago, Chicago, IL 60637, USA

${ }^{19}$ Dept. of Physics, University of Chicago, Chicago, IL 60637, USA

${ }^{20}$ Enrico Fermi Institute, University of Chicago, Chicago, IL 60637, USA

${ }^{21}$ Kavli Institute for Cosmological Physics, University of Chicago, Chicago, IL 60637, USA

22 Dept. of Physics and Astronomy, University of Canterbury, Private Bag 4800, Christchurch, New Zealand

${ }^{23}$ Dept. of Physics, University of Maryland, College Park, MD 20742, USA

${ }^{24}$ Dept. of Astronomy, Ohio State University, Columbus, OH 43210, USA

${ }^{25}$ Dept. of Physics and Center for Cosmology and Astro-Particle Physics, Ohio State University, Columbus, OH 43210, USA

${ }^{26}$ Niels Bohr Institute, University of Copenhagen, DK-2100 Copenhagen, Denmark

${ }^{27}$ Dept. of Physics, TU Dortmund University, D-44221 Dortmund, Germany

${ }^{28}$ Dept. of Physics and Astronomy, Michigan State University, East Lansing, MI 48824, USA

${ }^{29}$ Dept. of Physics, University of Alberta, Edmonton, Alberta, Canada T6G 2E1

${ }^{30}$ Erlangen Centre for Astroparticle Physics, Friedrich-Alexander-Universität Erlangen-Nürnberg, D-91058 Erlangen, Germany

${ }^{31}$ Physik-department, Technische Universität München, D-85748 Garching, Germany

${ }^{32}$ Département de physique nucléaire et corpusculaire, Université de Genève, CH-1211 Genève, Switzerland

${ }^{33}$ Dept. of Physics and Astronomy, University of Gent, B-9000 Gent, Belgium

${ }^{34}$ Dept. of Physics and Astronomy, University of California, Irvine, CA 92697, USA

${ }^{35}$ Karlsruhe Institute of Technology, Institute for Astroparticle Physics, D-76021 Karlsruhe, Germany

${ }^{36}$ Karlsruhe Institute of Technology, Institute of Experimental Particle Physics, D-76021 Karlsruhe, Germany

${ }^{37}$ Dept. of Physics, Engineering Physics, and Astronomy, Queen's University, Kingston, ON K7L 3N6, Canada

${ }^{38}$ Dept. of Physics and Astronomy, University of Kansas, Lawrence, KS 66045, USA

${ }^{39}$ Dept. of Physics and Astronomy, University of Nebraska-Lincoln, Lincoln, Nebraska 68588, USA

${ }^{40}$ Dept. of Physics, King's College London, London WC2R 2LS, United Kingdom

${ }^{41}$ School of Physics and Astronomy, Queen Mary University of London, London E1 4NS, United Kingdom

42 Department of Physics and Astronomy, UCLA, Los Angeles, CA 90095, USA

${ }^{43}$ Department of Physics, Mercer University, Macon, GA 31207-0001, USA

${ }^{44}$ Dept. of Astronomy, University of Wisconsin-Madison, Madison, WI 53706, USA

${ }^{45}$ Dept. of Physics and Wisconsin IceCube Particle Astrophysics Center, University of Wisconsin-Madison, Madison, WI 53706, USA

${ }^{46}$ Institute of Physics, University of Mainz, Staudinger Weg 7, D-55099 Mainz, Germany

${ }^{47}$ School of Physics and Astronomy, The University of Manchester, Oxford Road, Manchester, M13 9PL, United Kingdom

${ }^{48}$ Department of Physics, Marquette University, Milwaukee, WI, 53201, USA

${ }^{49}$ Institut für Kernphysik, Westfälische Wilhelms-Universität Münster, D-48149 Münster, Germany

${ }^{50}$ Bartol Research Institute and Dept. of Physics and Astronomy, University of Delaware, Newark, DE 19716, USA

${ }^{51}$ Dept. of Physics, Yale University, New Haven, CT 06520, USA

${ }^{52}$ Columbia Astrophysics and Nevis Laboratories, Columbia University, New York, NY 10027, USA

${ }^{53}$ Dept. of Physics, University of Notre Dame du Lac, 225 Nieuwland Science Hall, Notre Dame, IN 46556-5670, USA

${ }^{54}$ Dept. of Physics, University of Oxford, Parks Road, Oxford OX1 3PU, UK

${ }^{55}$ Dept. of Physics, Drexel University, 3141 Chestnut Street, Philadelphia, PA 19104, USA

${ }^{56}$ Physics Department, South Dakota School of Mines and Technology, Rapid City, SD 57701, USA

${ }^{57}$ Dept. of Physics, University of Wisconsin, River Falls, WI 54022, USA

${ }^{58}$ Dept. of Physics and Astronomy, University of Rochester, Rochester, NY 14627, USA

${ }^{59}$ Department of Physics and Astronomy, University of Utah, Salt Lake City, UT 84112, USA

${ }^{60}$ Oskar Klein Centre and Dept. of Physics, Stockholm University, SE-10691 Stockholm, Sweden

${ }^{61}$ Dept. of Physics and Astronomy, Stony Brook University, Stony Brook, NY 11794-3800, USA

${ }^{62}$ Dept. of Physics, Sungkyunkwan University, Suwon 16419, Korea

${ }^{63}$ Institute of Basic Science, Sungkyunkwan University, Suwon 16419, Korea

${ }^{64}$ Earthquake Research Institute, University of Tokyo, Bunkyo, Tokyo 113-0032, Japan

65 Dept. of Physics and Astronomy, University of Alabama, Tuscaloosa, AL 35487, USA

${ }^{66}$ Dept. of Astronomy and Astrophysics, Pennsylvania State University, University Park, PA 16802, USA

${ }^{67}$ Dept. of Physics, Pennsylvania State University, University Park, PA 16802, USA

${ }^{68}$ Institute of Gravitation and the Cosmos, Center for Multi-Messenger Astrophysics, Pennsylvania State University, University Park, PA 16802, USA 
${ }^{69}$ Dept. of Physics and Astronomy, Uppsala University, Box 516, S-75120 Uppsala, Sweden

${ }^{70}$ Dept. of Physics, University of Wuppertal, D-42119 Wuppertal, Germany

${ }^{71}$ DESY, D-15738 Zeuthen, Germany

72 Università di Padova, I-35131 Padova, Italy

${ }^{73}$ National Research Nuclear University, Moscow Engineering Physics Institute (MEPhI), Moscow 115409, Russia

\section{Acknowledgements}

USA - U.S. National Science Foundation-Office of Polar Programs, U.S. National Science Foundation-Physics Division, U.S. National Science Foundation-EPSCoR, Wisconsin Alumni Research Foundation, Center for High Throughput Computing (CHTC) at the University of Wisconsin-Madison, Open Science Grid (OSG), Extreme Science and Engineering Discovery Environment (XSEDE), Frontera computing project at the Texas Advanced Computing Center, U.S. Department of Energy-National Energy Research Scientific Computing Center, Particle astrophysics research computing center at the University of Maryland, Institute for Cyber-Enabled Research at Michigan State University, and Astroparticle physics computational facility at Marquette University; Belgium - Funds for Scientific Research (FRS-FNRS and FWO), FWO Odysseus and Big Science programmes, and Belgian Federal Science Policy Office (Belspo); Germany - Bundesministerium für Bildung und Forschung (BMBF), Deutsche Forschungsgemeinschaft (DFG), Helmholtz Alliance for Astroparticle Physics (HAP), Initiative and Networking Fund of the Helmholtz Association, Deutsches Elektronen Synchrotron (DESY), and High Performance Computing cluster of the RWTH Aachen; Sweden - Swedish Research Council, Swedish Polar Research Secretariat, Swedish National Infrastructure for Computing (SNIC), and Knut and Alice Wallenberg Foundation; Australia - Australian Research Council; Canada - Natural Sciences and Engineering Research Council of Canada, Calcul Québec, Compute Ontario, Canada Foundation for Innovation, WestGrid, and Compute Canada; Denmark - Villum Fonden and Carlsberg Foundation; New Zealand Marsden Fund; Japan - Japan Society for Promotion of Science (JSPS) and Institute for Global Prominent Research (IGPR) of Chiba University; Korea - National Research Foundation of Korea (NRF); Switzerland - Swiss National Science Foundation (SNSF); United Kingdom - Department of Physics, University of Oxford. 